\title{
Clinical associations between ASCT2 and p-mTOR in the pathogenesis and prognosis of epithelial ovarian cancer
}

\author{
HUIMING GUO $^{1^{*}}$, YU XU $^{2 *}$, FENG WANG $^{2}$, ZONGWEN SHEN $^{2}$, XIAOYU TUO $^{3}$, \\ HONG QIAN $^{1}$, HUAWEI WANG ${ }^{4}$ and KUNHUA WANG ${ }^{5,6}$ \\ Departments of ${ }^{1}$ Gynecology, ${ }^{2}$ Gastrointestinal Surgery, ${ }^{3}$ Pathology and ${ }^{4}$ Reproduction and Genetics, \\ The First Affiliated Hospital of Kunming Medical University; \\ ${ }^{5}$ Yunnan Engineering Technology Center of Diagnosis and Treatment of Digestive Diseases; \\ ${ }^{6}$ Department of Gastrointestinal Surgery, The First Affiliated Hospital of Kunming Medical University, \\ Kunming, Yunnan 650032, P.R. China
}

Received January 25, 2018; Accepted September 7, 2018

DOI: $10.3892 /$ or.2018.6729

\begin{abstract}
Alanine serine cysteine-preferring transporter 2 (ASCT2; also known as SLC1A5) is an important glutamine transporter, and it serves a crucial role in tumor growth and progression. ASCT2 is highly expressed in numerous types of cancer, but the pathological significance of its expression in epithelial ovarian cancer (EOC) remains unclear. The mechanistic target of rapamycin (mTOR) level is hyperelevated in a number of tumor types, including ovarian cancer. The aim of the present study was to elucidate the prognostic role of ASCT2 and phosphorylated (p)-mTOR in EOC. The levels of ASCT2 and $\mathrm{p}-\mathrm{mTOR} / \mathrm{mTOR}$ were detected in normal ovarian tissues, benign ovarian tumors, borderline ovarian tumors and EOC tissues by reverse transcription-quantitative polymerase chain reaction (RT-qPCR) and western blot assays. The protein levels of ASCT2 and p-mTOR in EOC patients were then detected by immunohistochemistry (IHC). Furthermore, EOC tumor sections were stained for Ki-67 and cluster of differentiation 34 (CD34) to assess proliferation and microvessel density by IHC. The results of RT-qPCR and western blot analysis demonstrated that ASCT2 and p-mTOR protein levels were significantly higher in EOC tissues compared with those in
\end{abstract}

Correspondence to: Professor Kunhua Wang, Yunnan Engineering Technology Center of Diagnosis and Treatment of Digestive Diseases, 295 Xichang Road, Kunming, Yunnan 650032, P.R. China E-mail: wangkunhuabio@163.com

Dr Huawei Wang, Department of Reproduction and Genetics, The First Affiliated Hospital of Kunming Medical University, 295 Xichang Road, Kunming, Yunnan 650032, P.R. China

E-mail: wanghuawei99@163.com

*Contributed equally

Key words: epithelial ovarian cancer, alanine serine cysteinepreferring transporter 2, phosphorylated-mechanistic target of rapamycin, Ki-67, cluster of differentiation 34 other groups. IHC analysis of 104 EOC tissues suggested that ASCT2 expression was associated with clinicopathological parameters, including International Federation of Gynecology and Obstetrics stage, pathological grade, serum cancer antigen 125 level, Ki-67 status and CD34 status. Kaplan-Meier survival curve analysis indicated that high expression of ASCT2 and p-mTOR were important factors predicting a poor prognosis for patients with EOC. The expression levels of ASCT2 and p-mTOR in EOC were positively correlated $(r=0.385, \mathrm{P}<0.001)$. This positive correlation between ASCT2 and $\mathrm{p}$-mTOR indicates that they have a synergistic effect on the growth and development of early EOC. The combined detection of ASCT2 and p-mTOR may serve as a potential marker to inform diagnosis, postoperative follow-up requirements and targeted therapy options for patients with early-stage EOC, but not for terminal-stage patients.

\section{Introduction}

Ovarian cancer is one of the most common gynecological malignancies and has numerous histological types; epithelial ovarian cancer (EOC) accounts for $85-90 \%$ of ovarian cancer cases (1). Due to the anatomical location of the ovary, early lesions are not easy to detect, which results in the diagnosis of the majority of ovarian cancer patients at a late stage (2). Ovarian cancer has the characteristics of early metastasis, recurrence and drug resistance; the 5-year survival rate of ovarian cancer is only $30-40 \%$, and the mortality rate is the highest among the gynecological malignancies (3). Therefore, it is of great importance to actively search for novel biomarkers to improve EOC diagnosis and prognosis.

Glutamine is an abundant non-essential amino acid in the human body, and a number of tumor cells consume much glutamine and show the 'glutamine dependence' phenomenon (4). Glutamine is transported by cellular transporters and is regarded as a precursor for the synthesis of numerous nucleotides, amino acids, proteins and other biologically important molecules; glutamine also provides glutathione and nicotinamide adenine dinucleotide phosphate to maintain redox homeostasis (5). Therefore, glutamine serves a crucial 
role in cell metabolism and proliferation. Aberrant bioenergetics is one of the markers of cancer cells (6). To satisfy rapid growth, cancer cells have to use another energy source, e.g., glutamine (7). A greater amount of glutamine is used for anabolic metabolism in cancer cells than is used in normal cells (8). Glutamine metabolism restriction can suppress cancer cell proliferation (9).

Glutamine must cross the plasma membrane to be used by cells, and this process requires specific transporters (10). There are a number of types of transporters used for transport across the plasma membrane, including $\mathrm{Na}^{+}$-dependent system $\mathrm{N}, \mathrm{Na}^{+}$-dependent system $\mathrm{A}, \mathrm{Na}^{+}$-independent system $\mathrm{L}$ and $\mathrm{Na}^{+}$-dependent system Alanine serine cysteine (ASC) (11). Moreover, the transporter differs between normal cells and tumor cells (12). The ASC system is commonly expressed in human cancer cells $(13,14)$. Alanine serine cysteine-preferring transporter 2 (ASCT2; also known as SLC1A5), belongs to the ASC system And acts as a high-affinity glutamine transporter in cancer cells (11). ASCT2 regulates the uptake of amino acids, including glutamine $(15,16)$. Numerous studies have reported that ASCT2 is closely associated with various cancer types, including breast cancer (17), clear-cell renal cell (18) and hepatocellular carcinoma (19), prostate (20), colorectal (21) and gastric cancer (22). In addition, downregulation of ASCT2 inhibits glutamine uptake, which successfully prevents tumor cell proliferation in prostate cancer (20), non-small cell lung cancer $(23,24)$, melanoma (25) and acute myeloid leukemia (26). However, studies involved in determining the expression and clinical significance of ASCT2 in EOC remain few in number.

Mechanistic target of rapamycin (mTOR) can be activated by the increased consumption of glutamine in cancer, which then promotes cell proliferation (27). In mammalian cells, mTOR has two complexes, mTORC1 and mTORC2 $(28,29)$. The mTOR pathway serves an important role in the progression of ovarian cancer (30). Blockade of ASCT2 decreases glutamine uptake through downregulation of the mTORC1 pathway in lymph node carcinoma in prostate cancer (20). However, the clinical significance of mTOR expression in EOC remains unclear.

The present study investigated the association between ASCT2 and p-mTOR expression and clinicopathological features in EOC, and the impact of the proteins ASCT2 and p-mTOR on the clinical factors, pathogenesis and prognosis of EOC. Moreover, the association between these proteins and the expression of $\mathrm{Ki}-67$ and CD34 in EOC was also evaluated.

\section{Materials and methods}

Patients. All methods were approved by the Research Medical Ethics Committee of Kunming Medical University (Kunming, Yunnan, China) and were performed in accordance with the approved guidelines. Patients were retrospectively analyzed from between April 18, 2007 and January 1, 2017, and these times were regarded as the start and end points for measuring survival.The inclusion criteria included the following: i) Patients with epithelial ovarian cancer who were diagnosed according to clinical manifestations and pathology, and for whom the clinicopathological data were complete; and ii) patients who did not receive radiotherapy and chemotherapy prior to surgery and who received combined chemotherapy consisting of Taxol and cisplatin (TP chemotherapy) following surgery. The exclusion criteria included the following: i) Patients pathologically diagnosed with reproductive cell tumors; ii) patients receiving neoadjuvant chemotherapy or radiotherapy prior to surgery; and iii) patients who received non-TP chemotherapy following surgery, or who did not receive regular chemotherapy. A total of 104 eligible tissues were collected from the patients at the First Affiliated Hospital of Kunming Medical University. Another 17 normal ovarian tissues, 14 benign ovarian tumor tissues, 19 borderline ovarian tumor tissues and 19 EOC tissues were used to obtain RNA and protein, and also were obtained from the First Affiliated Hospital of Kunming Medical University. The median age of these patients was 49 years, ranging from 16 to 71 years. For immunohistochemical, tissues were fixed with $10 \%$ formaldehyde, followed by dehydration and paraffin embedding, and storage at room temperature. For the reverse transcription-quantitative polymerase chain reaction (RT-qPCR) and western blotting, tissues were kept at $-80^{\circ} \mathrm{C}$ in a refrigerator. The clinical data of the patients, including age, International Federation of Gynecology and Obstetrics (FIGO) stage, tumor type, pathological grade and preoperative serum tumor marker values were collected. Tumor stages were histologically classified according to the 2010 American Joint Committee on Cancer Tumor-Node-Metastasis (TNM) classification (31).

$R T-q P C R$. The 17 normal ovarian tissues, 14 benign ovarian tumor tissues, 19 borderline ovarian tumor tissues and 19 EOC tissues were used to obtain RNA. RNA extraction was performed using an RNAprep Pure Tissue kit (Tiangen Biotech, Co., Ltd., Beijing, China) according to the manufacturer's protocols. RNA $(1 \mu \mathrm{g})$ was used for cDNA synthesis with a cDNA synthesis kit (Thermo Fisher Scientific, Inc., Waltham, MA, USA) following the manufacturer's protocols. qPCR was performed using TaqMan Fast Advanced Master mix (Thermo Fisher Scientific, Inc.) on an ABI 7500 thermal cycler (Applied Biosystems). The PCR content was as follows: $10 \mu \mathrm{l} 2 \mathrm{X}$ PCR Master mix, $1 \mu \mathrm{l}$ primers and probe, $1 \mu \mathrm{l} \mathrm{cDNA}$ and $\mathrm{ddH}_{2} \mathrm{O}$ up to a total reaction volume of $20 \mu \mathrm{l}$. The thermal cycling protocol was as follows: Initial denaturation at $95^{\circ} \mathrm{C}$ for $5 \mathrm{~min}$, and 20 cycles of denaturation at $95^{\circ} \mathrm{C}$ for $10 \mathrm{~min}$ and annealing and extension at $60^{\circ} \mathrm{C}$ for $60 \mathrm{sec}$. The $\beta$-actin gene was used as a house-keeping gene. The relative mRNA expression was calculated by the $2^{-\Delta \Delta C q}$ method (32). The primers and TaqMan probe sequences for ASCT2 and $\beta$-actin were as follows: ASCT2 forward, CTCCTTGATCCTGGCTGTGG, reverse, GGGCAGCTCACTCTTCACTT and probe, CCG TCCTCAATGTAGAAGGTGACGC; $\beta$-actin forward GCC AACACAGTGCTGTCT, reverse, GGAGCAATGATCTTG ATCTT and probe, TCACCAACTGGGACGACATGGAGA AA. The primer sequences for mTOR were as follows: mTOR forward, CCAACAGTTCACCCTCAGGT and reverse, GCT GCCACTCTCCAAGTTTC.

Western blot assay. Total protein was extracted by radioimmunoprecipitation assay lysis buffer (Beyotime Institute of Biotechnology, Shanghai, China). The concentrations of protein were determined using a bicinchoninic acid protein assay kit (Beyotime Institute of Biotechnology). A total of 
$30 \mu \mathrm{g}$ protein per lane was loaded on $10 \%$ SDS-PAGE gels and then transferred to a polyvinylidene fluoride membrane and blocked with $10 \%$ skimmed milk in Tris-buffered saline plus Tween-20 for $2 \mathrm{~h}$ at room temperature. Subsequent to blocking, membranes were immunoblotted with rabbit anti-ASCT2 antibody (1:1,000 dilution; cat. no. bs-0473R), anti-p-mTOR antibody (Ser2481) (1:1,000 dilution; cat. no. bs-3495R), anti-mTOR antibody (1:1,000 dilution; cat. no. bs-1992R) and anti-GAPDH antibody (1:5,000 dilution; cat. no. bs-10900R) (all Bioss, Beijing, China), as appropriate, at $4^{\circ} \mathrm{C}$ overnight, and were then incubated with HRP-labeled goat anti-rabbit secondary antibodies (1:2,000 dilution; cat. no. bs-0295G; Bioss) for $1 \mathrm{~h}$ at room temperature. The blotted protein bands were visualized using Pierce ${ }^{\mathrm{TM}}$ ECL Western Blotting Substrate (Thermo Fisher Scientific, Inc.). The optical density of the resulting bands was determined by Image $\mathrm{J} 2 \mathrm{x}$ software (Rawak Software, Inc., Dresden, Germany) (33), with normalization of the densitometry measures to GAPDH.

Immunohistochemistry (IHC). Sections (5- $\mu$ m thick) were cut from paraffin samples. Sections were dewaxed with ethanol and xylene, and were then washed 3 times in phosphate-buffered saline (PBS). Next, antigen repair was performed on the sections with $0.01 \mathrm{M}$ citric acid buffer ( $\mathrm{pH} 6.0)$ at $100^{\circ} \mathrm{C}$ temperature and $80 \mathrm{kpa}$ pressure. Sections were blocked by incubation with $5 \%$ goat serum in PBS for $15 \mathrm{~min}$ at room temperature. The sections were then incubated with anti-ASCT2 antibody (1:100 dilution; cat. no. bs-0473R), anti-p-mTOR antibody (Ser2481) (1:100 dilution; cat. no. bs-3495R), anti-CD34 antibody (1:100 dilution; catalog no. bs-0646R) and anti-Ki-67 antibody (1:100 dilution; cat. no. bs-23102R) (all Bioss), as appropriate, and then with horseradish peroxidase-conjugated goat anti-rabbit antibody (1:200 dilution; cat. no. bs-0295G; Bioss). Staining was detected in the sections using a DAB kit (MXB Biotechnologies, Fuzhou, China) at room temperature for 3-10 min, and the sections were then observed under a microscope (Nikon Corporation, Tokyo, Japan). The percentage of positive cells was scored as follows: 0, no positive cells; $1,1-25 \%$ positive cells; $2,26-50 \%$ positive cells; $3,51-75 \%$ positive cells; and 4,76-100\% positive cells. The intensity was estimated as follows: 0 , negative; 1 , weak; 2 , moderate; and 3 , strong. The percentage and intensity scores were multiplied; a total score of $<6$ was considered as low expression, while a score of $\geq 7$ was considered as high expression.

Statistics. Differences in mRNA and protein expression were calculated by one-way analysis of variance, followed by Tukey's post hoc test. The survival curve was generated using the Kaplan-Meier technique and differences between these curves were analyzed by the log-rank test. Factors that had prognostic significance in the univariate analysis were further analyzed using a multivariate Cox regression model. Associations between ASCT2/p-mTOR expression and clinicopathological characteristics were analyzed by the $\chi^{2}$ test. Correlations between the expression of ASCT2 and p-mTOR were analyzed by Spearman's correlation analysis. For all tests, $\mathrm{P}<0.05$ was considered to indicate a statistically significant difference. All statistical data were analyzed using SPSS 13.11 software (SPSS. Inc., Chicago, IL, USA).
Table I. Clinical characteristics of patients with epithelial ovarian cancer.

\begin{tabular}{|c|c|}
\hline Characteristics & Value \\
\hline Total patients, n (\%) & $104(100.00)$ \\
\hline \multicolumn{2}{|l|}{ Age at surgery, years } \\
\hline Mean & 49.39 \\
\hline \multicolumn{2}{|l|}{ FIGO stage, n (\%) } \\
\hline Stage I & $32(30.77)$ \\
\hline Stage II & $18(17.31)$ \\
\hline Stage III & $45(43.27)$ \\
\hline Stage IV & $9(8.65)$ \\
\hline \multicolumn{2}{|l|}{ Tumor type, n (\%) } \\
\hline Serous & $45(43.27)$ \\
\hline Mucinous & $19(18.27)$ \\
\hline Endometrioid & $25(24.04)$ \\
\hline Clear cell & $12(11.54)$ \\
\hline Others & $3(2.88)$ \\
\hline \multicolumn{2}{|c|}{ Pathological grade, n (\%) } \\
\hline $\mathrm{I}$ & $41(39.42)$ \\
\hline II & $43(41.35)$ \\
\hline III & $18(17.31)$ \\
\hline IV & $2(1.92)$ \\
\hline \multicolumn{2}{|l|}{$\mathrm{Ki}-67, \mathrm{n}(\%)$} \\
\hline Low & $68(65.38)$ \\
\hline High & $36(34.62)$ \\
\hline \multicolumn{2}{|l|}{ CD34, n (\%) } \\
\hline Positive & $13(12.50)$ \\
\hline Negative & $91(87.50)$ \\
\hline
\end{tabular}

CD34, cluster of differentiation; FIGO, International Federation of Gynecology and Obstetrics.

\section{Results}

mRNA and protein expression of ASCT2 in normal ovarian tissues and EOC tissues. The mRNA and protein expression of ASCT2 was analyzed by qPCR and western blotting, respectively, in normal ovarian tissues, benign ovarian tumors, borderline ovarian tumors and EOC tissues. ASCT2 mRNA and protein levels were markedly higher in ovarian tumor tissues than in normal ovarian tissues, and were highest in EOC tissues compared with that in benign ovarian tumor and borderline ovarian tumor tissues (Fig. 1A and B). p-mTOR protein level was markedly higher in EOC tissues compared with that in normal ovarian tissues, benign ovarian tumors and borderline ovarian tumor tissues (Fig. 1C). These results suggested that the ASCT2 and p-mTOR levels in EOC are the highest compared with that in other tissues. Thus, EOCs were used for subsequent analysis of the association between ASCT2/p-mTOR and EOC.

Clinicopathological characteristics of patients with EOC. A total of 104 patients with EOC were analyzed. The clinicopathological characteristics of the 104 EOC patients are summarized in Table I. The median age of the patients was 
Table II. Association between ASCT2/p-mTOR expression and clinicopathological parameters.

\begin{tabular}{|c|c|c|c|c|c|c|c|}
\hline \multirow[b]{2}{*}{ Characteristics } & \multirow[b]{2}{*}{$\mathrm{n}$} & \multicolumn{2}{|c|}{ ASCT2 } & \multirow[b]{2}{*}{ P-value ${ }^{a}$} & \multicolumn{2}{|c|}{ p-mTOR } & \multirow[b]{2}{*}{ P-value ${ }^{a}$} \\
\hline & & Low & High & & Low & High & \\
\hline Age at surgery, years & & & & & & & 0.072 \\
\hline Mean & 49.39 & 50.11 & 48.33 & 0.265 & 50.72 & 45.42 & \\
\hline FIGO stage, $n$ & & & & & & & $<0.001$ \\
\hline Stage I & 32 & 26 & 6 & $<0.001$ & 29 & 3 & \\
\hline Stage II & 18 & 12 & 6 & & 15 & 3 & \\
\hline Stage III & 45 & 23 & 22 & & 32 & 13 & \\
\hline Stage IV & 9 & 1 & 8 & & 2 & 7 & \\
\hline Tumor type, $\mathrm{n}$ & & & & & & & 0.006 \\
\hline Serous & 45 & 24 & 21 & 0.190 & 29 & 16 & \\
\hline Mucinous & 19 & 10 & 9 & & 13 & 6 & \\
\hline Endometrioid & 25 & 18 & 7 & & 22 & 3 & \\
\hline Clear cell & 12 & 10 & 2 & & 11 & 1 & \\
\hline Others & 3 & 0 & 3 & & 3 & 0 & \\
\hline Pathological grade, $\mathrm{n}$ & & & & & & & 0.426 \\
\hline I & 41 & 19 & 22 & 0.026 & 29 & 12 & \\
\hline II & 43 & 28 & 15 & & 33 & 10 & \\
\hline III & 18 & 15 & 3 & & 3 & 3 & \\
\hline IV & 2 & 0 & 2 & & 1 & 1 & \\
\hline Serum CA125, n & & & & & & & 0.001 \\
\hline Mean & 129.98 & 89.17 & 334.83 & $<0.001$ & 98.24 & 319.75 & \\
\hline Ki-67, n & & & & & & & 0.004 \\
\hline Low & 68 & 48 & 20 & 0.002 & 57 & 11 & \\
\hline High & 36 & 14 & 22 & & 21 & 15 & \\
\hline CD34, n & & & & & & & 0.001 \\
\hline Positive & 13 & 3 & 10 & 0.004 & 5 & 8 & \\
\hline Negative & 91 & 59 & 32 & & 73 & 18 & \\
\hline
\end{tabular}

${ }^{a} \chi^{2}$ test. ASCT2, alanine serine cysteine-preferring transporter 2; p-mTOR, phosphorylated-mechanistic target of rapamycin; CA125, cancer antigen 125; FIGO, International Federation of Gynecology and Obstetrics.

49 years, ranging from 14 to 80 years. The tumor types of the patients included 45 serous adenocarcinoma, 19 mucinous adenocarcinoma, 25 endometrioid adenocarcinoma, 12 clear cell adenocarcinoma and 3 other histological types (Table I). The day of surgery was considered as the start day for measuring postoperative survival.

ASCT2/p-mTOR protein expression and the association with the clinicopathological characteristics of patients with EOC. ASCT2 and p-mTOR levels were detected in 104 epithelial ovarian tumor tissues by IHC assay. The 104 patients with EOC were divided into the low-expression group and high-expression group according to the level of ASCT2 and p-mTOR expression. As shown in Fig. 2A, the ASCT2 protein was localized mainly on the plasma membrane. The p-mTOR protein was localized mainly in the cytoplasm (Fig. 2). The ASCT2 percentage scores in the high-expression and low-expression patient groups were $59.62 \%(62 / 104)$ and $40.38 \%$ (42/104), respectively. The p-mTOR percentage scores in the high-expression and low-expression patient groups were
$75.00 \%$ (78/104) and 25.00\% (26/104), respectively (Table II). The association between ASCT2 or p-mTOR status and clinicopathological characteristics, respectively, was also analyzed (Table II). As shown in Table II, the expression of ASCT2 and p-mTOR was significantly associated with high FIGO stage $(\mathrm{P}<0.001)$, and the presence and concentration of serum cancer antigen $125(\mathrm{P}<0.01), \mathrm{Ki}-67$ status $(\mathrm{P}<0.01)$ and CD34 status $(\mathrm{P}<0.01)$. However, neither ASCT2 expression nor p-mTOR expression was significantly associated with age. It was also found that the expression of $\mathrm{p}$-mTOR was significantly associated with tumor type $(\mathrm{P}=0.006)$ and that the expression of ASCT2 was significantly associated with pathological grade $(\mathrm{P}=0.026)$ (Table II).

Furthermore, the correlation analysis of ASCT2 expression and p-mTOR expression suggested a positive correlation between these two proteins in EOC patients $(r=0.385$, $\mathrm{P}<0.001$ ) (Table III).

Prognostic value of ASCT2/p-mTOR expression in EOC. Univariate analysis was used to evaluate ASCT2/p-mTOR 

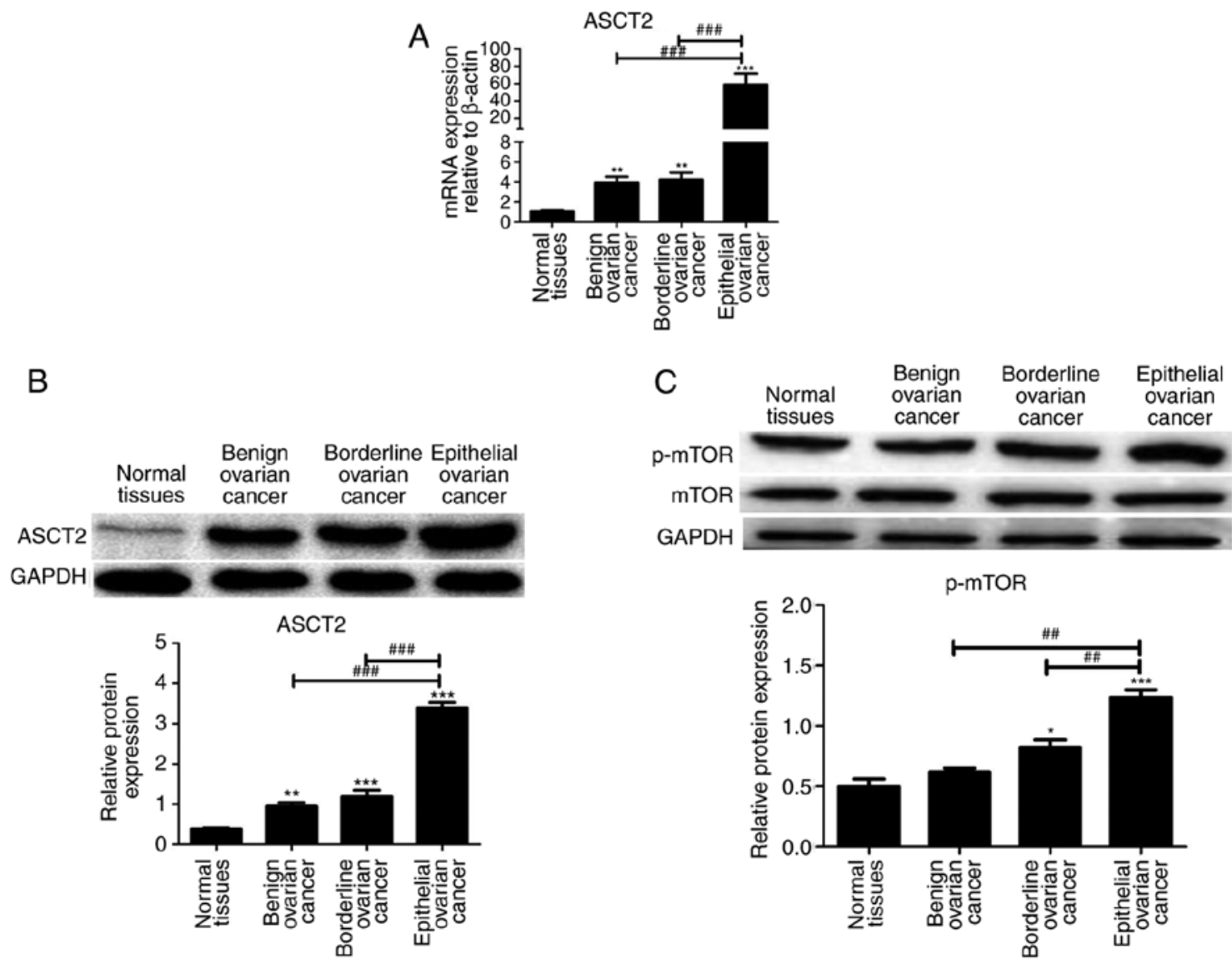

Figure 1. ASCT2 and p-mTOR expression levels were detected by reverse transcription-quantitative polymerase chain reaction and/or western blot assay in normal ovarian tissues and EOC tissues. (A) The ASCT2 mRNA expression levels in EOC tissues and normal tissues. (B) The ASCT2 protein expression levels and quantitative analysis in EOC tissues and normal tissues. (C) The p-mTOR protein expression levels and quantitative analysis in EOC tissues and normal tissues. mRNA data was normalized to $\beta$-actin and protein data was normalized to GAPDH. Data are presented as the mean \pm standard deviation of 3 independent experiments. ${ }^{*} \mathrm{P}<0.05,{ }^{* * *} \mathrm{P}<0.01$ and ${ }^{* * * *} \mathrm{P}<0.001 ;{ }^{\# \#} \mathrm{P}<0.01$ and ${ }^{\# \# \#} \mathrm{P}<0.001$. ASCT2, alanine serine cysteine-preferring transporter 2; p-mTOR, phosphorylated-mechanistic target of rapamycin; EOC, epithelial ovarian cancer.

Table III. Correlation between the expression of ASCT2 and p-mTOR.

\begin{tabular}{lrrrrrr}
\hline & \multicolumn{3}{c}{ ASCT2 } & & \\
\cline { 2 - 4 } p-mTOR & Low & High & Total & r & P-value $^{\text {a }}$ \\
\hline Low & 55 & 7 & 62 & 0.385 & $<0.001$ \\
High & 23 & 19 & 42 & & \\
Total & 78 & 26 & 104 & & \\
\hline
\end{tabular}

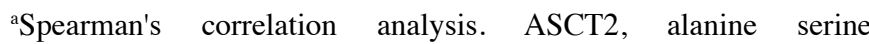
cysteine-preferring transporter 2; p-mTOR, phosphorylated-mechanistic target of rapamycin.

protein expression and other clinicopathological parameters for their prognostic value in EOC. High expression of ASCT2, high expression of p-mTOR, high FIGO stage, high serum CA125 level, high Ki-67 level and high CD34 status were significantly associated with poor overall survival. These parameters were unfavorable predictors for overall survival of EOC patients (Table IV). Subsequently, these prognostic factors were further analyzed by multivariate Cox proportional hazards regression model analysis. This analysis revealed that high expression of ASCT2 [hazard ratio (HR), 2.062; $\mathrm{P}=0.036]$, high expression of $\mathrm{p}-\mathrm{mTOR}$ (HR, 0.377; $\mathrm{P}=0.015$ ), FIGO stage (HR, 2.329; $\mathrm{P}<0.001)$ and Ki-67 status (HR, 2.111; $\mathrm{P}=0.018$ ) were prognostic markers of overall survival of $\mathrm{EOC}$ patients (Table IV).

Kaplan-Meier survival curves of patients with positive and negative ASCT2/p-mTOR expression were generated. These curves indicated that the patient survival rate was significantly associated with ASCT2/p-mTOR expression. The association between ASCT2 protein expression and overall survival was analyzed and it was found that patients with low ASCT2 $(\mathrm{P}<0.001)$ or $\mathrm{p}$-mTOR $(\mathrm{P}=0.046)$ expression experienced a significantly longer overall survival time compared with that of patients with high ASCT2 or p-mTOR expression (Fig. 3A and B). In addition, patients with high Ki-67 expression had a poorer overall survival rate $(\mathrm{P}<0.001)$ than patients with low Ki-67 expression (Fig. 3C), and patients in FIGO stage IV had a lower overall survival rate $(\mathrm{P}<0.001)$ than patients in TNM stages I-III (Fig. 3D).

As shown in Fig. 4A, the overall survival rate of patients with low expression of ASCT2 and p-mTOR was longer than patients with high expression of either ASCT2 or p-mTOR $(\mathrm{P}=0.036)$ in early EOC patients. Importantly, it was also found that the 


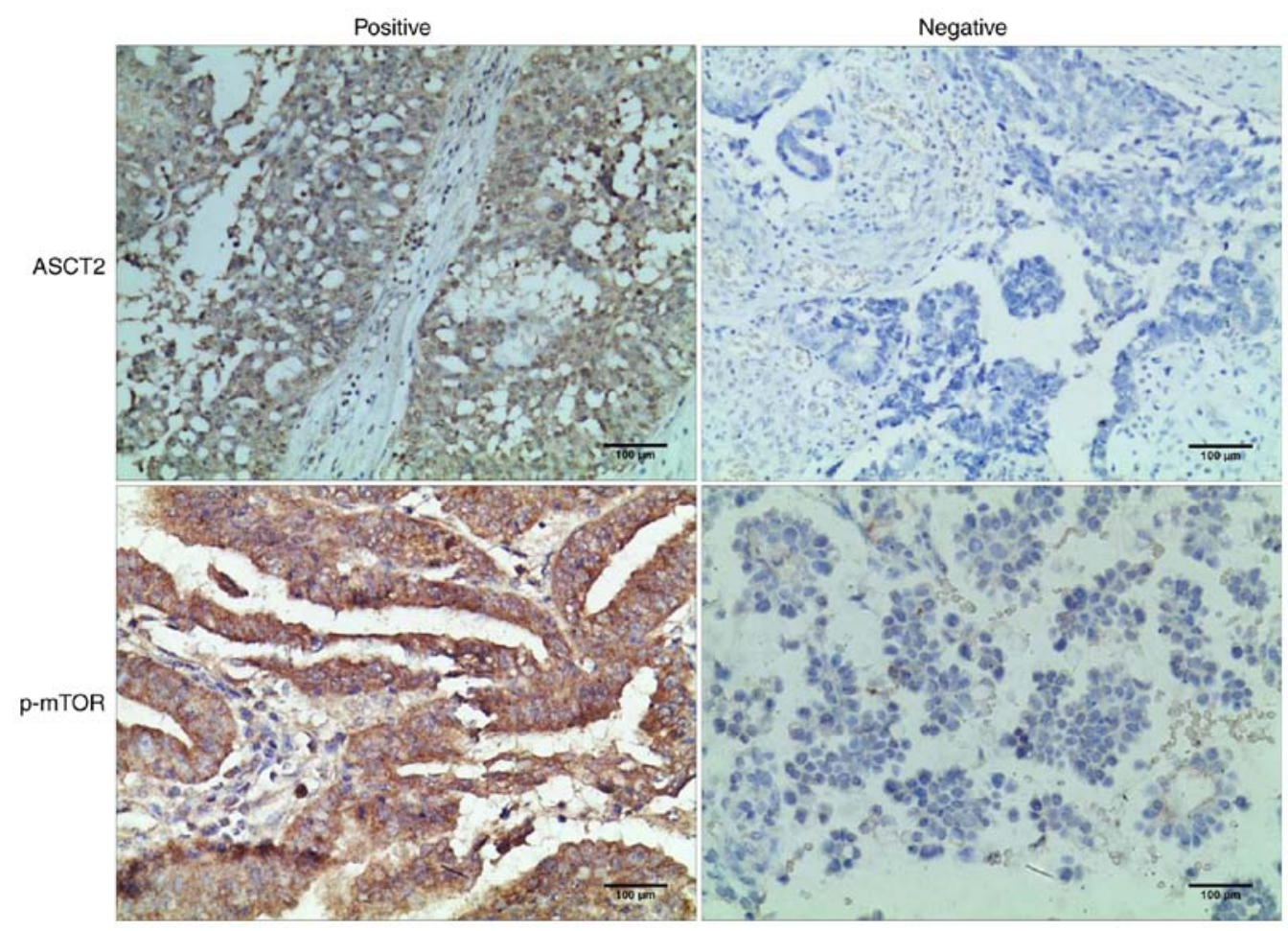

Figure 2. Immunohistochemical staining of tumor tissue from epithelial ovarian cancer. Representative images with x20 magnification are shown. ASCT2 exhibited a membranous immunostaining pattern. p-mTOR exhibited a cytoplasmic immunostaining pattern. Scale bar, $100 \mu \mathrm{m}$. ASCT2, alanine serine cysteine-preferring transporter 2; p-mTOR, phosphorylated-mechanistic target of rapamycin.

A

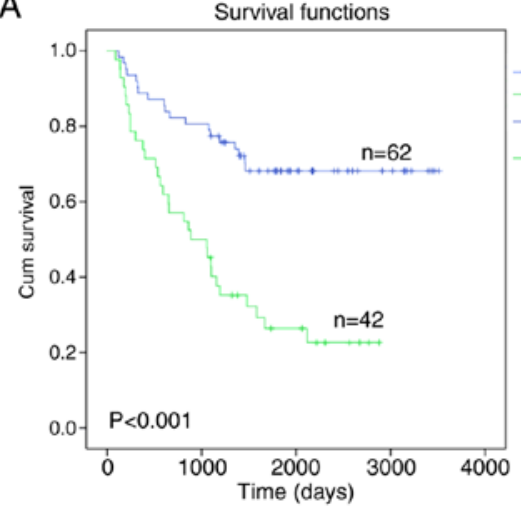

C

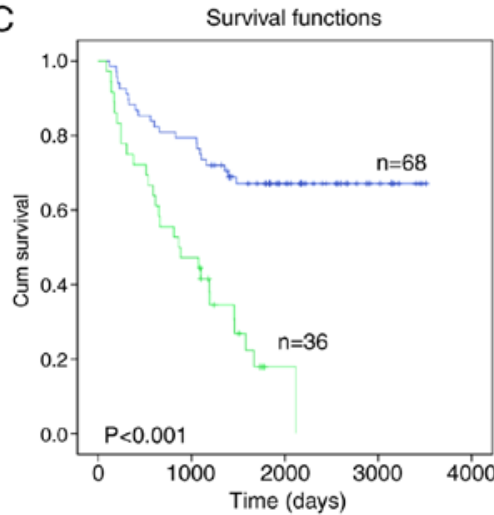

B ASCT2
Low expression
High expression
Low expression-
censored
High expression
censored

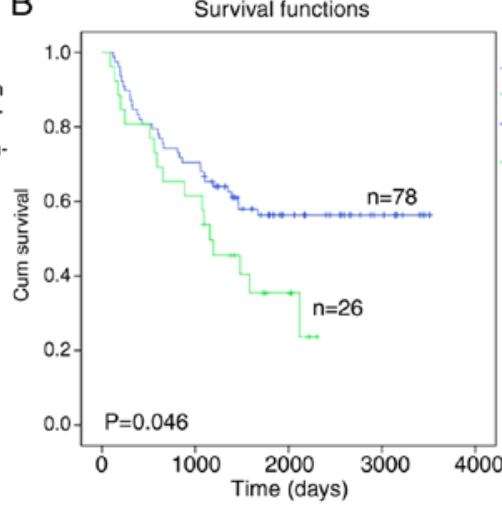

p-mTOR

Low expression Low expressionHigh expression.

D
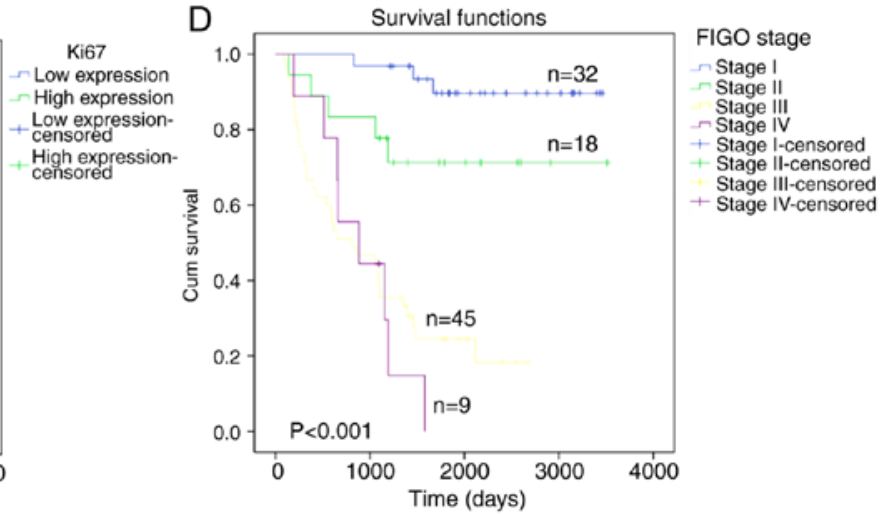

Figure 3. Survival analysis of EOC patients by Kaplan-Meier analysis. (A) The overall survival rate of EOC patients with high expression of ASCT2 (green line) was significantly lower than that in EOC patients with low ASCT2 expression (blue line). (B) The overall survival rate of EOC patients with high expression of p-mTOR (green line) was statistically lower than that of EOC patients with low p-mTOR expression (blue line). (C) The overall survival rate of EOC patients with high expression of Ki-67 (green line) was statistically lower than that of EOC patients with low Ki-67 expression (blue line). (D) The overall survival rate of EOC patients with advanced TNM stage IV (purple line) was significantly lower than that in EOC patients with TNM stage I-III (blue, green and yellow lines). EOC, epithelial ovarian cancer; ASCT2, alanine serine cysteine-preferring transporter 2; p-mTOR, phosphorylated-mechanistic target of rapamycin; TNM, Tumor-Node-Metastasis; FIGO, International Federation of Gynecology and Obstetrics. 
Table IV. Univariate and multivariate analysis of overall survival in patients with epithelial ovarian cancer.

\begin{tabular}{|c|c|c|c|c|}
\hline \multirow[b]{2}{*}{ Variable } & \multirow{2}{*}{$\frac{\text { Univariate analysis }}{\text { P-value }^{\mathrm{a}}}$} & \multicolumn{3}{|c|}{ Multivariate analysis } \\
\hline & & $\mathrm{HR}$ & $95 \% \mathrm{CI}$ & P-value ${ }^{b}$ \\
\hline Age at surgery, years & ns & & & \\
\hline FIGO stage & $<0.001$ & 2.329 & $1.536-3.529$ & $<0.001$ \\
\hline Tumor type & ns & & & \\
\hline Pathological grade & ns & & & \\
\hline Serum ca125 & $<0.001$ & & & ns \\
\hline Ki-67 & $<0.001$ & 2.111 & $1.136-3.924$ & 0.018 \\
\hline CD34 & $<0.001$ & & & ns \\
\hline ASCT2 & $<0.001$ & 2.062 & $1.050-4.048$ & 0.036 \\
\hline p-mTOR & 0.046 & 0.377 & $0.172-0.827$ & 0.015 \\
\hline
\end{tabular}

${ }^{\mathrm{a}}$ Log-rank test; ${ }^{\mathrm{b}} \mathrm{Cox}$ proportional hazard regression model. CI, confidence interval; ns, not significant.
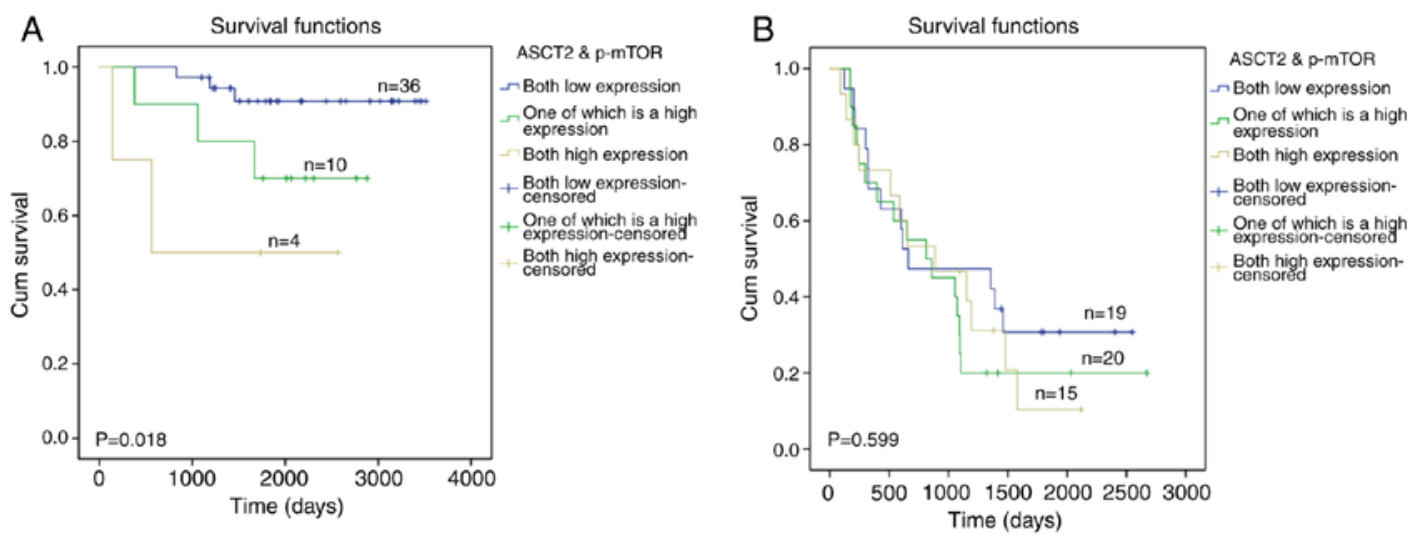

Figure 4. Kaplan-Meier survival curves for EOC patients by ASCT2/p-mTOR expression level. (A) The overall survival rate of early EOC patients with high expression of ASCT2 and p-mTOR (brown line) was significantly lower than that of early EOC patients with low expression of ASCT2 and p-mTOR (blue line) or high expression of either ASCT2 or p-mTOR (green line). (B) There were no significant differences with regard to the overall survival rates among the patients with high expression of ASCT2 and p-mTOR (brown line), low expression of ASCT2 and p-mTOR (blue line) and high expression of either ASCT2 or p-mTOR (green line) in the terminal-stage patients with EOC. EOC, epithelial ovarian cancer; ASCT2, alanine serine cysteine-preferring transporter 2; p-mTOR, phosphorylated-mechanistic target of rapamycin.

overall survival rate of patients with high expression of ASCT2 and p-mTOR was shorter than patients with low expression of ASCT2 and p-mTOR $(\mathrm{P}<0.001)$ and patients with high expression of either ASCT2 or $\mathrm{p}$-mTOR $(\mathrm{P}=0.024)$ in patients with early-stage EOC. However, as shown in Fig. 4B, there was no significant difference between the groups with high expression of ASCT2 and p-mTOR or with high expression of either ASCT2 or p-mTOR $(\mathrm{P}=0.599)$ in the patients with terminal-stage EOC. These results indicated that the combined detection of ASCT2 and p-mTOR may serve as a potential marker to inform diagnosis and targeted therapy options for early EOC patients but not for terminal EOC patients.

\section{Discussion}

Amino acid metabolism and the involved transporters are crucial for the proliferation and growth of cancer cells (34). ASCT2, an important glutamine transporter has been reported to be upregulated in numerous cancer types, including breast cancer, clear cell renal cell carcinoma and lung cancer $(17,18,35,36)$. ASCT2 levels are correspondingly increased in cancer (11). It was reported that the activation of mTOR was associated with adverse prognostic factors in EOC $(37,38)$. Elevated ASCT2 expression promoted cell growth and survival through mTOR signaling in lung cancer (23). However, the association between ASCT2 and p-mTOR proteins, and the clinical significance of this association in patients with EOC remains unknown. To the best of our knowledge, the present clinicopathological study is the first to investigate the clinical significance of ASCT2 and p-mTOR, and the association between them, in patients with EOC.

The present results showed that ASCT2 was highly expressed in EOC tissues compared with that in normal, benign and borderline ovarian cancer tissues. However, a previous study reported that ASCT2 expression was upregulated in the majority of EOC cases, and that there was no significant difference between EOC tissue and borderline malignancy (39). One important cause for this difference between the present study and the previous study may be that the southwest of China is contains more areas with ethnic 
minorities, thus there are certain differences in ethnicity. Our future research will focus on the influence of differences in ethnicity in EOC patients.

In the present study, the expression of ASCT2 was associated with pathological variables, including cell proliferation (Ki-67), angiogenesis (angiogenic markers, microvessel density as determined by CD34 expression) and the activation of the mTOR signaling pathway. It was found that the expression levels of ASCT2 and p-mTOR were significantly associated with FIGO stage $(\mathrm{P}<0.001)$, serum CA125 concentration $(\mathrm{P}<0.01)$, Ki-67 status $(\mathrm{P}<0.01)$ and $\mathrm{CD} 34$ status $(\mathrm{P}<0.01)$. However, neither ASCT2 expression nor $\mathrm{p}-\mathrm{mTOR}$ expression were significantly associated with age.

These findings suggest that ASCT2 and the mTOR signaling pathway are activated and probably serve an important role in the development of EOC. Although Kaira et al (39) reported that ASCT2 was highly expressed in ovarian tumors, indicating that ASCT2 is associated with the prognosis of ovarian tumors, the present study focused on the prognostic significance of ASCT2 in EOC and evaluated the associations between ASCT2 and p-mTOR in EOC.

In a previous report, glutamine promoted ovarian cancer cell proliferation through the mTOR/S6 pathway, suggesting a close association between amino acid metabolism and the mTOR signaling pathway (40). ASCT2 mediates the uptake of glutamine in tumors. In the present study, it was found that ASCT2 expression was positively associated with p-mTOR expression. Using Kaplan-Meier survival curve analysis, either high expression of ASCT2 or high expression of p-mTOR was significant associated with poor overall survival. Furthermore, it was found that there was a positive correlation between ASCT2 expression and p-mTOR expression in EOC patients. The Kaplan-Meier survival curves for EOC patients by ASCT2/p-mTOR expression showed that patients with higher co-expression of ASCT2/p-mTOR experienced shorter survival times than patients with low co-expression and patients with high expression of either ASCT2 or p-mTOR in the early-stage patients, but this association was not true for terminal-stage patients with EOC. This finding indicated that ASCT2 and p-mTOR may play a mutual role in EOC, as demonstrated by the marked correlation between ASCT2 and p-mTOR expression levels in early-stage patients with EOC.

In summary, the present study demonstrated the association between ASCT2/p-mTOR expression levels and the prognosis of patients with EOC. Although the high expression of ASCT2 or p-mTOR alone was identified as a significant prognostic predictor, the coexpression of ASCT2 and p-mTOR was a more powerful indicator for predicting worse outcome in early-stage patients with EOC. This study suggests that ASCT2 and p-mTOR expression may be promising prognostic biomarkers and therapeutic targets in early-stage EOC.

\section{Acknowledgements}

Not applicable.

\section{Funding}

This project was supported by funding from the Yunling Scholar Foundation of the First Affiliated Hospital of
Kunming Medical University (grant no. 2016BS001), the Key Science and Technology Planning Project of Kunming Science and Technology Bureau and the Key Science and Technology Planning Project of Yunnan Provincial Science and Technology Department (grant no. 2016FC005).

\section{Availability of data and materials}

All data generated or analyzed during this study are included in this published article and are freely available to any researchers.

\section{Authors' contributions}

HW, KW and HG conceived and designed the experiments; YX, FW, ZS, XT and HQ performed the experiments and analyzed the data; and YX wrote the manuscript. All authors have read and approved the final manuscript.

\section{Ethics approval and consent to participate}

All human tissue samples were collected by the First Affiliated Hospital of Kunming Medical University and written informed consent was obtained from all patients. All methods were approved by the Research Medical Ethics Committee of Kunming Medical University and were performed in accordance with the approved guidelines.

\section{Patient consent for publication}

Not applicable.

\section{Competing interests}

The authors declare that they have no competing interests.

\section{References}

1. Morgan RJ Jr, Alvarez RD, Armstrong DK, Boston B, Burger RA, Chen LM, Copeland L, Crispens MA, Gershenson D, Gray HJ, et al: Epithelial ovarian cancer. J Natl Compr Canc Netw 9: 82-113, 2011.

2. Prat J: Ovarian carcinomas: Five distinct diseases with different origins, genetic alterations, and clinicopathological features. Virchows Arch 460: 237-249, 2012.

3. Siegel R, Ward E, Brawley O and Jemal A: Cancer statistics, 2011: The impact of eliminating socioeconomic and racial disparities on premature cancer deaths. CA Cancer J Clin 61: 212-236, 2011.

4. Hensley CT, Wasti AT and DeBerardinis RJ: Glutamine and cancer: Cell biology, physiology, and clinical opportunities. J Clin Invest 123: 3678-3684, 2013.

5. Mohamed A, Deng X, Khuri FR and Owonikoko TK: Altered glutamine metabolism and therapeutic opportunities for lung cancer. Clin Lung Cancer 15: 7-15, 2014.

6. Hanahan D and Weinberg RA: Hallmarks of cancer: The next generation. Cell 144: 646-674, 2011.

7. Zhdanov AV, Waters AH, Golubeva AV, Dmitriev RI and Papkovsky DB: Availability of the key metabolic substrates dictates the respiratory response of cancer cells to the mitochondrial uncoupling. Biochim Biophys Acta 1837: 51-62, 2014.

8. Gonzalez Herrera KN, Lee $\mathbf{J}$ and Haigis MC: Intersections between mitochondrial sirtuin signaling and tumor cell metabolism. Crit Rev Biochem Mol Biol 50: 242-255, 2015.

9. Fuchs BC and Bode BP: Stressing out over survival: Glutamine as an apoptotic modulator. J Surg Res 131: 26-40, 2006. 
10. Medina MA,Sánchez-JimenezF,MárquezJ,RodriguezQuesada A and Nuñez de Castro I: Relevance of glutamine metabolism to tumor cell growth. Mol Cell Biochem 113: 1-15, 1992.

11. Fuchs BC and Bode BP: Amino acid transporters ASCT2 and LAT1 in cancer: Partners in crime? Semin Cancer Biol 15: 254-266, 2005.

12. Dolinska M, Dybel A, Zablocka B and Albrecht J: Glutamine transport in C6 glioma cells shows ASCT2 system characteristics. Neurochem Int 43: 501-507, 2003.

13. Kilberg MS, Stevens BR and Novak DA: Recent advances in mammalian amino acid transport. Annu Rev Nutr 13: 137-165, 1993.

14. McGivan JD and Pastor-Anglada M: Regulatory and molecular aspects of mammalian amino acid transport. Biochem J 299 321-334, 1994.

15. Kanai Y and Hediger MA: The glutamate/neutral amino acid transporter family SLC1: Molecular, physiological and pharmacological aspects. Pflugers Arch 447: 469-479, 2004.

16. Kekuda R, Prasad PD, Fei YJ, Torres-Zamorano V, Sinha S, Yang-Feng TL, Leibach FH and Ganapathy V: Cloning of the sodium-dependent, broad-scope, neutral amino acid transporter Bo from a human placental choriocarcinoma cell line. J Biol Chem 271: 18657-18661, 1996.

17. van Geldermalsen $M$, Wang $Q$, Nagarajah R, Marshall AD, Thoeng A, Gao D, Ritchie W, Feng Y, Bailey CG, Deng N, et al: ASCT2/SLC1A5 controls glutamine uptake and tumour growth in triple-negative basal-like breast cancer. Oncogene 35: 3201-3208, 2016

18. Liu Y, Yang L, An H, Chang Y, Zhang W, Zhu Y, Xu L and Xu J: High expression of solute carrier family 1 , member 5 (SLC1A5) is associated with poor prognosis in clear-cell renal cell carcinoma. Sci Rep 5: 16954, 2015.

19. Sun HW, Yu XJ, Wu WC, Chen J, Shi M, Zheng L and Xu J: GLUT1 and ASCT2 as predictors for prognosis of hepatocellular carcinoma. PLoS One 11: e0168907, 2016.

20. Wang Q, Hardie RA,Hoy AJ, van Geldermalsen M, Gao D, Fazli L, Sadowski MC, Balaban S, Schreuder M, Nagarajah R, et al: Targeting ASCT2-mediated glutamine uptake blocks prostate cancer growth and tumour development. J Pathol 236: 278-289, 2015.

21. Toda K, Nishikawa G, Iwamoto M, Itatani Y, Takahashi R, Sakai Y and Kawada K: Clinical role of ASCT2 (SLC1A5) in KRAS-mutated colorectal cancer. Int J Mol Sci 18: pii: E1632, 2017.

22. Kasai N, Sasakawa A, Hosomi K, Poh TW, Chua BL, Yong WP, So J, Chan SL, Soong R, Kono K, et al: Anti-tumor efficacy evaluation of a novel monoclonal antibody targeting neutral amino acid transporter ASCT2 using patient-derived xenograft mouse models of gastric cancer. Am J Transl Res 9: 3399-3410, 2017.

23. Hassanein M, Hoeksema MD, Shiota M, Qian J, Harris BK, Chen H, Clark JE, Alborn WE, Eisenberg R and Massion PP SLC1A5 mediates glutamine transport required for lung cancer cell growth and survival. Clin Cancer Res 19: 560-570, 2013.

24. Hassanein M, Qian J, Hoeksema MD, Wang J, Jacobovitz M, Ji X, Harris FT, Harris BK, Boyd KL, Chen H, et al: Targeting SLCla5-mediated glutamine dependence in non-small cell lung cancer. Int J Cancer 137: 1587-1597, 2015.
25. Wang Q, Beaumont KA, Otte NJ, Font J, Bailey CG, van Geldermalsen M, Sharp DM, Tiffen JC, Ryan RM, Jormakka M, et al: Targeting glutamine transport to suppress melanoma cell growth. Int J Cancer 135: 1060-1071, 2014.

26. Willems L, Jacque N, Jacquel A, Neveux N, Maciel TT, Lambert M, Schmitt A, Poulain L, Green AS, Uzunov M, et al: Inhibiting glutamine uptake represents an attractive new strategy for treating acute myeloid leukemia. Blood 122: 3521-3532, 2013.

27. Wise DR and Thompson CB: Glutamine addiction: A new therapeutic target in cancer. Trends Biochem Sci 35: 427-433, 2010.

28. Montero JC, Chen X, Ocaña A and Pandiella A: Predominance of mTORC1 over mTORC2 in the regulation of proliferation of ovarian cancer cells: Therapeutic implications. Mol Cancer Ther 11: 1342-1352, 2012.

29. Zoncu R, Efeyan A and Sabatini DM: mTOR: From growth signal integration to cancer, diabetes and ageing. Nat Rev Mol Cell Biol 12: 21-35, 2011.

30. Dobbin ZC and Landen CN: The importance of the $\mathrm{PI} 3 \mathrm{~K} / \mathrm{AKT} / \mathrm{MTOR}$ pathway in the progression of ovarian cancer. Int J Mol Sci 14: 8213-8227, 2013.

31. Edge SB and Compton CC: The American Joint Committee on Cancer: The 7th edition of the AJCC cancer staging manual and the future of TNM. Ann Surg Oncol 17: 1471-1474, 2010.

32. Livak KJ and Schmittgen TD: Analysis of relative gene expression data using real-time quantitative PCR and the $2^{-\triangle \Delta C \mathrm{~T}}$ method. Methods 25: 402-408, 2001.

33. Gallo-Oller G, Ordoñez R and Dotor J: A new background subtraction method for Western blot densitometry band quantification through image analysis software. J Immunol Methods 457: $1-5,2018$.

34. Hammoudi N, Ahmed KB, Garcia-Prieto C and Huang P: Metabolic alterations in cancer cells and therapeutic implications. Chin J Cancer 30: 508-525, 2011.

35. Huang F, Zhao Y, Zhao J, Wu S, Jiang Y, Ma $\mathrm{H}$ and Zhang T: Upregulated SLC1A5 promotes cell growth and survival in colorectal cancer. Int J Clin Exp Pathol 7: 6006-6014, 2014.

36. Shimizu K, Kaira K, Tomizawa Y, Sunaga N, Kawashima O, Oriuchi N, Tominaga H, Nagamori S, Kanai Y, Yamada M, et al: ASC amino-acid transporter 2 (ASCT2) as a novel prognostic marker in non-small cell lung cancer. Br J Cancer 110: 2030-2039, 2014.

37. Noske A, Lindenberg JL, Darb-Esfahani S, Weichert W, Buckendahl AC, Röske A, Sehouli J, Dietel M and Denkert C: Activation of mTOR in a subgroup of ovarian carcinomas: Correlation with p-eIF-4E and prognosis. Oncol Rep 20 1409-1417, 2008.

38. No JH, Jeon YT, Park IA, Kim YB, Kim JW, Park NH, Kang SB, Han JY, Lim JM and Song YS: Activation of mTOR signaling pathway associated with adverse prognostic factors of epithelial ovarian cancer. Gynecol Oncol 121: 8-12, 2011.

39. Kaira $K$, Nakamura $K$, Hirakawa $T$, Imai $H$, Tominaga $H$, Oriuchi N, Nagamori S, Kanai Y, Tsukamoto N, Oyama T, et al: Prognostic significance of L-type amino acid transporter 1 (LAT1) expression in patients with ovarian tumors. Am J Transl Res 7: 1161-1171, 2015.

40. Yuan L, Sheng X, Willson AK, Roque DR, Stine JE, Guo H, Jones HM, Zhou C and Bae-Jump VL: Glutamine promotes ovarian cancer cell proliferation through the mTOR/S6 pathway. Endocr Relat Cancer 22: 577-591, 2015. 